\title{
Avaliação das Propriedades Química, Física e Mecânica de Painéis Aglomerados Produzidos com Resíduo de Madeira da Amazônia - Cumaru (Dipteryx Odorata) e Resina Poliuretana à Base de Óleo de Mamona
}

\author{
Mírian Dayse Lima Zau, Raimundo Pereira de Vasconcelos, Virginia Mansanares Giacon \\ Faculdade de Tecnologia - FT, Universidade Federal do Amazonas - UFAM \\ Francisco Antônio Rocco Lahr \\ Escola de Engenharia de São Carlos - EESC, Universidade de São Paulo - USP
}

\begin{abstract}
Resumo: O beneficiamento de toras de madeira produz uma grande quantidade de resíduos, em torno de $60 \%$, os quais em geral são aproveitados na geração de energia com a produção de cinzas e fuligem, ou dispostos em locais inadequados, gerando um problema ambiental sério. Nesse sentido, o presente estudo tem como objetivo principal produzir e avaliar física e mecanicamente painéis produzidos com resíduos do beneficiamento de madeira de Dipteryx odorata (Cumaru), espécie tropical cuja caracterização química e física também foi realizada. Para a produção dos painéis fez-se uso de um planejamento fatorial $\left(2^{2}+1\right)$ no qual foram analisadas as quantidades de resíduo e resina. Para a análise físico-mecânica, foram produzidos 24 painéis em diferentes condições de quantidade de resíduo (1000; $1300 ; 1500 \mathrm{~g})$ e teor de resina $(10 ; 12,5$ e $15 \%)$. Os painéis foram cortados para obtenção dos corpos-de-prova para determinação de densidade, razão de compactação, inchamento e absorção ( 2 e 24 h); módulo de ruptura e de elasticidade em flexão estática, adesão interna e arrancamento de parafuso. Os painéis a $1500 \mathrm{~g}(10$ e $15 \%)$ foram os que apresentaram os melhores resultados mecânicos, superiores ao mínimo especificado pela norma NBR 14810-3 (2006), evidenciando seu potencial na produção desses painéis.
\end{abstract}

Palavras-chave: Resíduos de madeira, painéis aglomerados, resina poliuretana à base de óleo de mamona, propriedades quimicas e mecânicas.

\section{Chemical, Physical and Mechanical Properties of Particleboard Produced with Amazon Wood Waste - Cumaru (Dipteryx Odorata) - and Castor Oil Based Polyurethane Adhesive}

\begin{abstract}
Logs processing produce large quantity of waste, ca. $60 \%$ related to original solid volume. This material is usually employed in power generation with the production of ash and soot, or improperly deposed, causing environmental problems. In this context, the present study aims to produce and evaluate the physical and mechanical performance of particleboards produced with waste from the processing of Dipteryx odorata, a tropical wood species whose chemical and physical characterization was also performed. The production of particleboards was based on a factorial design $\left(2^{2}\right.$ +1 ), in which the quantities of waste and resin were analyzed. For the physical and mechanical analysis, twenty-four panels were produced, using different amount of residues (1000, 1300, $1500 \mathrm{~g})$ and resin content $(10,12.5 \mathrm{and} 15 \%)$. Specimens were obtained from the panels in order to determine their: density; compaction ratio, thickness swelling and water absorption ( 2 and $24 \mathrm{~h}$ ); modulus of rupture and modulus of elasticity in static bending; internal adhesion; and screw pullout. Panels with $1500 \mathrm{~g}$ (10 and $15 \%$ of resin) were those with the best mechanical results, higher than the minimum specified by the NBR 14810-3 (2006), confirming their potential in producing particleboards.
\end{abstract}

Keywords: Wood waste, particleboards, castor oil based polyurethane adhesive, physical, chemical and mechanical properties.

\section{Introdução}

O termo resíduo de madeira pode ser associado à palavra problema, pois geralmente a sua disposição ou utilização adequada gera custos elevados. Porém, o conhecimento da quantidade, da qualidade e das possibilidades de uso deste material pode gerar uma alternativa de uso que viabilize o seu manuseio ${ }^{[1]}$.

Dentre os vários tipos de espécies de madeira encontradas na região amazônica, podemos citar o
Cumaru (Dipteryx odorata), que é uma madeira nobre, cujas características são diferenciadas das espécies comuns, apresentando alta resistência estrutural e alta densidade $\left(0,95 \text { a } 1,00 \mathrm{~g} / \mathrm{cm}^{3}\right)^{[2]}$. Esta é uma espécie amplamente utilizada na indústria madeireira, porém, no seu beneficiamento, observa-se uma grande geração de resíduos, em torno de $60 \%$, que acabam sendo reaproveitados para geração de energia elétrica, através da

Autor para correspondência: Mírian Dayse Lima Záu, Programa de Pós-graduação em Engenharia Civil - PPGEC, Universidade Federal do Amazonas - UFAM, Avenida General Rodrigo Otávio, 6200, Coroado I, CEP 69077-000, Manaus, AM, Brasil, e-mail: mirian_dayse@ hotmail.com 
queima, conforme observado em visita realizada na empresa Mil Madeiras Preciosas Ltda, localizada no município de Itacoatiara, Km 227 da rodovia Manaus - Itacoatiara, Amazonas.

Porém, existem alternativas para o reaproveitamento dos resíduos descartados durante o processamento mecânico da madeira, como por exemplo, utilizá-los na fabricação de painéis aglomerados através do método de prensagem. A fabricação de painéis é ecologicamente correta, pois os resíduos irão transformar-se em produtos úteis, assim conservando os recursos naturais ${ }^{[3]}$.

No Brasil, as principais espécies utilizadas na produção de painéis aglomerados e MDF (medium density fiberboard) são dos gêneros Pinus e Eucalipto, as quais são plantadas em grande escala nas regiões sul e sudeste do país, em que estão localizadas as indústrias moveleiras. Nas regiões norte, nordeste e centro-oeste do país, além da ausência de pólos moveleiros que demandam grande quantidade de painéis, os plantios florestais em escala comercial ainda são restritos a pequenas áreas ${ }^{[4]}$.

Para a produção de painéis aglomerados se faz necessário o uso de uma resina com função aglomerante. As resinas mais comumente empregadas na produção de aglomerados são aquelas à base de uréia-formaldeído. Esses tipos de resinas apresentam dois inconvenientes: baixa resistência à ação de umidade e quando aquecida ocorre emanação de formaldeído, prejudicial ao meio ambiente. Com base nisto, há a necessidade de encontrar um substituto com igual qualidade e desempenho às resinas tradicionais ${ }^{[5]}$.

Nesse contexto, o presente estudo tem como objetivo produzir painéis utilizando resíduos de madeira nobre da Amazônia, com resina poliuretana (PU) em parte renovável, derivada do óleo da mamona (ricinus communis) que tem sido estudada há vários anos ${ }^{[6]}$ e que tem mostrado grande potencial na produção de compósitos lignocelulósicos ${ }^{[7-9]}$.

\section{Materiais e Métodos}

Para a produção dos painéis foram utilizados resíduos de Cumaru doados pela empresa Mil Madeiras Preciosas Ltda, localizada no município de Itacoatiara - Amazonas e a resina bicomponente à base de óleo de mamona doadas pela Empresa Plural Química Ltda, situada na cidade de São Carlos-São Paulo. As análises químicas como teor de extrativos (T204 cm-97), teor de lignina klason (T222 om-02) e teor de cinzas (T211 om-02), foram realizadas no Instituto de Química de São Carlos (IQSC-SP) baseadas na norma TAPPI (Technical Association of the Pulp and Paper Industry). O teor de lignina solúvel foi analisado de acordo com a metodologia ${ }^{[10]}$ e o teor de celulose e polioses foi determinado por análise cromatográfica no Cromatógrafo SHIMADZU, modelo CR 7A, utilizando o filtrado obtido na determinação do teor de lignina klason ${ }^{[11]}$.
Para a análise física de densidade básica da madeira, utilizou-se o método de medição direta do volume com o auxílio de paquímetro digital Mitutoyo $0,01 \mathrm{~mm}$ e o teor de umidade foi obtido através do equipamento OHAUS MB200 por meio de medição da porcentagem da perda de massa a $105^{\circ} \mathrm{C}$ por 180 minutos.

Os ensaios para a determinação das propriedades dos painéis foram realizados de acordo com a norma brasileira $^{[12]}$, que cita especificações para painéis de alta densidade.

Para a produção dos painéis optou-se por estudar as proporções de resíduo e resina através de um planejamento experimental Fatorial $2^{2}+1$ (Factorial Experimental Design - FED) ${ }^{[13]}$, de acordo com a Tabela 1. Para verificar a existência de diferenças significativas entre as variáveis, foram utilizados os testes $\mathrm{te} \mathrm{F}$ e a metodologia de superfície de resposta (curvas de nível) na análise da influência das variáveis de processo sobre a variável resposta.

Foram produzidos 4 corpos-de-prova para cada nível (superior e inferior) e 12 para o ponto central, totalizando 32 corpos-de-prova.

O processo de produção dos painéis iniciou-se com o trituramento do material no equipamento TR 500 e em seguida foram picados em moinho Marconi MA 680 a uma granulometria de 40 mesh $(0,42 \mathrm{~mm})$. A pressão, temperatura e tempo de prensagem foram de 5,0 $\mathrm{MPa}$, $95 \pm 5^{\circ} \mathrm{C}$ e 10 minutos, respectivamente, e o tempo de cura foi de 48 horas

\section{Resultados e Discussão}

\section{Propriedades físicas e químicas do resíduo}

O teor de umidade do Cumaru analisado foi de 10,2\%. Alguns autores afirmam que a umidade ideal de resíduo de madeira deve permanecer entre 8 a $12 \%^{[14.15]}$, para obtenção de melhor desempenho em painéis utilizando a resina da mamona. Porém Maloney ${ }^{[16]}$ afirma que a umidade dessas partículas deve situar-se em torno de 5\%. Assim, fez-se a secagem ao ar livre do resíduo, controlando diariamente seu teor de umidade, até ter atingido o valor 5,6\%. Ainda que a umidade das partículas tenha ficado fora do intervalo recomendado usualmente destacado para o uso da resina da mamona, os resultados foram superiores aos requisitos normativos.

A determinação da densidade básica foi obtida a partir dos resultados da massa seca e do volume úmido realizada em triplicata em corpos-de-prova de dimensões nominais de $3 \times 3 \times 3 \mathrm{~cm}^{3}$. Os resultados são apresentados na Tabela 2 e foram próximos aos resultados de Gonzaga ${ }^{[17]}$, característicos de espécie de alta densidade.

As análises químicas foram realizadas em triplicata e o teor de cinzas encontrado foi de 1,72\% $\pm 0,15$ (Tabela 2). Silva et al. ${ }^{[18]}$ analisou o Cumaru, utilizando a norma ASTM

Tabela 1. Fatores e níveis de planejamento experimental $2^{2}+1$

\begin{tabular}{lccc}
\hline & Nível inferior $(-\mathbf{1 )}$ & Ponto central (0) & Nível Superior (+1) \\
\hline Quantidade de resíduos (g) & 1000 & 1300 & 1500 \\
Quantidade de resina (\%) & 10 & 12,5 & 15 \\
\hline
\end{tabular}


$1102-56$ e encontrou valores de $0,3 \%$ para teor de cinzas, abaixo dos valores encontrados neste trabalho.

O teor de extrativos orgânicos encontrado foi de $18,32 \% \pm 1,24$ (Tabela 2), cujo resultado foi acima do encontrado por Santana e Okino ${ }^{[19]}(9,7 \%)$ que analisou a mesma espécie. Porém, esses teores (cinzas e extrativos) podem variar de acordo com as características do solo.

De acordo com Lima et al. ${ }^{[20]}$, a quantidade e o tipo de extrativo presente na madeira, podem interferir nas reações de polimerização da resina, influenciando na qualidade dos painéis produzidos. Madeiras com maior massa específica podem apresentar tilos ou impregnações por extrativos diminuindo assim a sua permeabilidade ${ }^{[21]}$. Com base nisso, pode inferir que a quantidade de resina para painéis com $1000 \mathrm{~g}$ de resíduos não foi suficiente para envolver todas as partículas, diminuindo a resistência mecânica dos painéis.

A lignina insolúvel apresentou média de 34,29\% \pm 0,26 . As análises químicas de lignina solúvel analisadas por espectroscopia na região ultravioleta apresentaram uma média de $0,014 \mathrm{~g} / \mathrm{L}(1,43 \%)$.

Pesquisas realizadas por Hillig ${ }^{[22]}$ demonstraram através de análises, que as forças de coesão e adesão das fibras aumentam com o decréscimo do conteúdo de lignina. Porém Gouvêa ${ }^{[23]}$, que produziu briquetes com resíduos de madeira da indústria moveleira adicionando lignina extraída do licor negro kraft, do gênero Eucalipto, encontrou resultados mecânicos superiores aos briquetes sem adição de lignina.

Os teores de celulose e polioses encontrados por intermédio de análise cromatográfica de alto desempenho (Tabela 2) apresentaram média de 46,80\% $\pm 1,42$ e 11,53\% $\pm 0,50$, respectivamente.

A porcentagem de celulose obtida nesta análise está de acordo com Klock et al. ${ }^{[24]}$, ou seja, para composições médias de madeiras folhosas. Já o teor de polioses encontrado apresentou média de $11,53 \%$, abaixo do valor citado por Klock et al. ${ }^{[24]}$ de $30 \%$ e Gonzaga ${ }^{[17]}$ que cita o teor de polioses de $20 \%$ a $28 \%$ nos tecidos das folhosas. É provável que a hemicelulose tenha sido degradada em parte com ácido sulfúrico na análise de teor de lignina insolúvel, já que as polioses sofrem forte ataque por ácidos e é solúvel, conforme Gonzaga ${ }^{[17]}$.

Os percentuais lignocelulósicos (teor de cinzas, extrativos, lignina solúvel e insolúvel, celulose e polioses) frequentemente ultrapassam $100 \%$, uma vez que cada análise é realizada com amostras diferentes, ou seja, a amostra usada para análise de lignina não é a mesma usada na análise de celulose, implicando em percentual total muitas vezes superior a $100 \%$.

\section{Propriedades físicas dos painéis}

Os resultados de densidade aparente nos painéis de Cumaru apresentaram características de alta densidade variando de $0,82 \mathrm{~g} / \mathrm{cm}^{3}$ (painéis com $1000 \mathrm{~g}$ a $10 \%$ ) a $1,01 \mathrm{~g} / \mathrm{cm}^{3}$ (painéis com $1500 \mathrm{~g}$ a $15 \%$ ). Os painéis produzidos com $1500 \mathrm{~g}$ de resíduos a 10 e $15 \%$ tenderam para maiores valores de densidade aparente (Tabela 3), por serem compostos por maior quantidade de partículas para o mesmo volume de painéis. Os resultados do planejamento estatístico indicaram que a variável resíduo foi significativa a $95 \%$ de confiança e suas variáveis dependentes foram conjuntamente significantes.

A razão de compactação é um fator importante na análise dos resultados, no entanto, a razão ideal indicada por Moslemi $^{[25]}$ e Maloney ${ }^{[26]}$ varia de 1,3 a 1,6 e não se aplica a espécies de madeira com alta densidade, conforme se verifica nos estudos realizados por Dias ${ }^{[15]}$, onde foram obtidos bons resultados com uma razão de compactação abaixo do intervalo indicado.

Os painéis de Cumaru apresentaram as mesmas características de Dias ${ }^{[15]}$, obtendo resultados mecânicos satisfatórios (Tabela 3) com uma RC menor à citada pelos autores anteriormente. Os dados estatísticos foram significativos para a variável resíduo e a análise de variância conjunta (ANOVA) apresentou grau de correlação de $\mathrm{R}^{2}=0,87$. As curvas de nível (Figura $1 \mathrm{~b}$ ) indicam que a resina

Tabela 2. Valores médios de densidade básica da madeira $\left(\mathrm{g} / \mathrm{cm}^{3}\right)$, teor de cinzas $(\%)$, teor de extrativos orgânicos $(\%)$, teor de lignina klason (\%), celulose (\%) e polioses $(\%)$.

\begin{tabular}{cccccccc}
\hline Amostras & $\begin{array}{c}\text { Densidade } \\
\text { básica }\left(\mathbf{g} / \mathbf{c m}^{\mathbf{3}}\right)\end{array}$ & $\begin{array}{c}\text { Teor de cinzas } \\
\mathbf{( \% )}\end{array}$ & $\begin{array}{c}\text { Teor de } \\
\text { Extrativos } \\
\text { orgânicos } \mathbf{( \% )}\end{array}$ & $\begin{array}{c}\text { Teor de } \\
\text { Lignina klason } \\
\mathbf{( \% )}\end{array}$ & $\begin{array}{c}\text { Teor de } \\
\text { Lignina solúvel } \\
\mathbf{( \% )}\end{array}$ & $\begin{array}{c}\text { Celulose }^{*} \\
\mathbf{( \% )}\end{array}$ & $\begin{array}{c}\text { Polioses }^{* * *} \\
\mathbf{( \% )}\end{array}$ \\
\hline $\mathrm{C} 1$ & 0,868 & 1,79 & 17,83 & 34,01 & 1,28 & 45,76 & 11,15 \\
$\mathrm{C} 2$ & 0,855 & 1,82 & 19,73 & 34,52 & 1,53 & 48,42 & 12,10 \\
C3 & 0,872 & 1,55 & 17,41 & 34,33 & 1,49 & 46,21 & 11,33 \\
Média & $0,865 \pm 0,009$ & $1,72 \pm 0,15$ & $18,32 \pm 1,24$ & $34,29 \pm 0,26$ & $1,43 \pm 0,13$ & $46,80 \pm 1,42$ & $11,53 \pm 0,50$ \\
\hline
\end{tabular}

$(*)$ (celobiose, glicose, ácido fórmico, furfural), $\left.{ }^{* *}\right)$ (ácido glucourônico, xilose, arabinose, ácido acético, furfural).

Tabela 3. Valores médios de densidade aparente $\left(\mathrm{g} / \mathrm{cm}^{3}\right), \mathrm{RC}$, teor de umidade (\%), inchamento em espessura (\%) e absorção de água (\%).

\begin{tabular}{|c|c|c|c|c|c|c|c|c|}
\hline \multicolumn{2}{|c|}{ Condições de processo } & \multirow{2}{*}{$\begin{array}{l}\text { Densidade } \\
\text { aparente } \\
\left(\mathrm{g} / \mathrm{cm}^{3}\right)\end{array}$} & \multirow{2}{*}{ RC } & \multirow{2}{*}{$\begin{array}{c}\text { Teor de } \\
\text { umidade }(\%)\end{array}$} & \multicolumn{2}{|c|}{$\begin{array}{c}\text { Inchamento em espessura } \\
(\%)\end{array}$} & \multicolumn{2}{|c|}{ Absorção de água (\%) } \\
\hline Resíduo (g) & Resina (\%) & & & & $2 \mathrm{~h}$ & $24 h$ & $2 \mathrm{~h}$ & $24 \mathrm{~h}$ \\
\hline 1000 & 10 & 0,82 & 0,95 & 5,91 & 0,73 & 4,05 & 2,44 & 7,72 \\
\hline 1000 & 15 & 0,82 & 0,95 & 5,36 & 0,77 & 2,71 & 2,25 & 6,02 \\
\hline 1300 & 12,5 & $0,97 \pm 0,09$ & 1,13 & $7,45 \pm 0,49$ & $0,95 \pm 1,01$ & $3,46 \pm 0,96$ & $1,84 \pm 0,39$ & $6,15 \pm 1,33$ \\
\hline 1500 & 10 & 1,00 & 1,16 & 5,97 & 0,58 & 2,61 & 1,93 & 6,00 \\
\hline 1500 & 15 & 1,01 & 1,17 & 5,29 & 0,70 & 2,11 & 2,11 & 4,39 \\
\hline
\end{tabular}




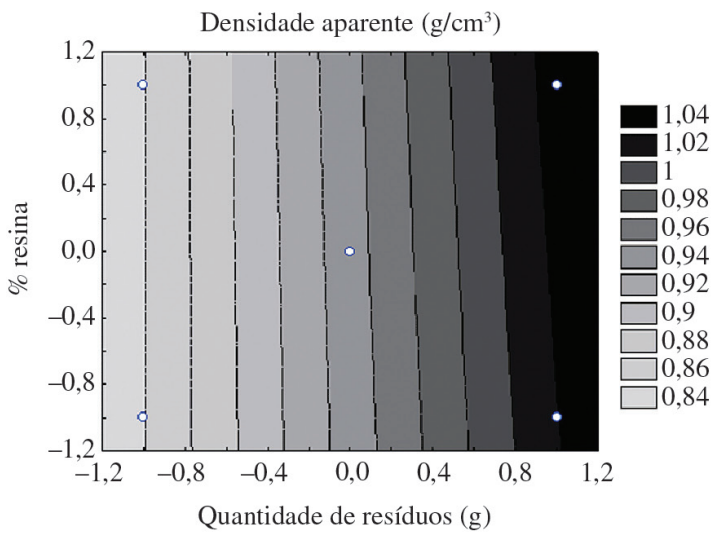

(a)

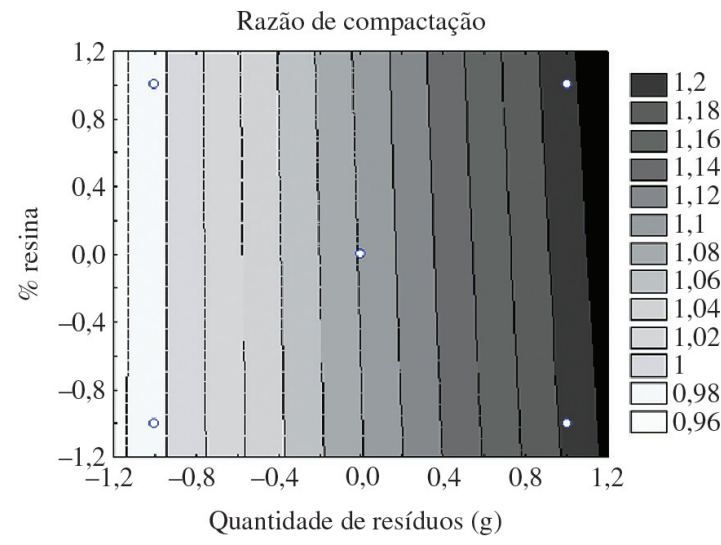

(b)

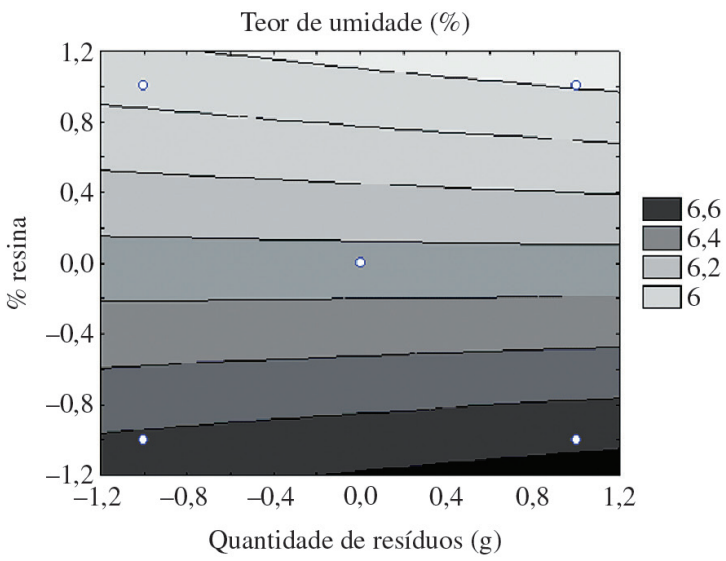

(c)

Figura 1. Curvas de nível para a variável resposta densidade aparente (a), RC (b) e Teor de Umidade (c) dos painéis de Cumaru. Quantidade de resíduos: $(-1=1000$ gramas $),(0=1300$ gramas $),(+1=1500$ gramas $) \%$ Resina: $(-1=10 \%),(0=12,5 \%),(+1=15 \%)$.

não interfere significativamente na variável resposta e a RC adquire maior valor com quantidade de resíduos maiores, nas condições estudadas.

O Teor de Umidade nos painéis de Cumaru a 1000 e $1500 \mathrm{~g}$ (Tabela 3) apresentaram resultados próximos, em torno de 5-6\%, com exceção dos painéis de $1300 \mathrm{~g}$ que apresentaram teor de umidade de 7,4\%. O tratamento estatístico indicou que os efeitos das variáveis não foram significativos nestas condições de processo. A representação da curva de nível (Figura 1c) indica que painéis com maiores porcentagens de resina apresentam menor teor de umidade, o que provavelmente está relacionado com a resina poliuretana do óleo da mamona que apresenta características de hidrofobicidade.

$\mathrm{O}$ resultado de inchamento em 2 horas dos painéis de Cumaru (Tabela 3) foi satisfatório conforme a norma ${ }^{[27]}$ que cita inchamento máximo a $8 \%$. Os resultados encontrados com painéis de Cumaru em 2 horas foram menores ao encontrado por Iwakiri et al. ${ }^{[28]}$ para painéis produzidos com resíduos de Piquiarana (espécie de madeira tropical, com densidade mais próxima ao Cumaru e coletada na mesma região) aderidos com resina uréia-formaldeído. Santos ${ }^{[7]}$ estudou painéis com fibras de curauá e resíduos de madeira tropical utilizando a resina da mamona e encontrou média de $6,2 \%$, valor próximo aos encontrados nesse trabalho. A norma brasileira ${ }^{[27]}$ não cita especificação para inchamento em espessura em 24 horas, porém os valores obtidos (Tabela 3) apresentaram-se inferiores aos encontrados por Iwakiri et al. ${ }^{[28]}$ com painéis de Piquiarana.

Com relação às análises de inchamento, verificou-se que o modelo adotado apresentou baixa relação linear entre as variáveis estudadas. Sendo assim, para estes resultados não foi realizado o tratamento estatístico.

Os painéis de Cumaru apresentaram valores de absorção de água entre $1-2 \%$ em 2 h (Tabela 3), resultados menores ao encontrado por Iwakiri et al. ${ }^{[28]}$ de $11,83 \%$ e Santos ${ }^{[7]}$ de $8,7 \%$. A absorção em 24 horas variou de 4 a $7 \%$, valores também menores aos encontrados com a Piquiarana $(28,30 \%)^{[28]}$. A baixa absorção nos painéis estudados provavelmente deve-se à característica hidrofóbica da resina utilizada.

O resultado estatístico de absorção em 2 horas indicou que a variável resíduo e a interação (resíduo e resina) foram significativas nas condições estudadas, enquanto a variável individual resina não foi significativa a $95 \%$ de confiança. As curvas de nível (Figura 2a) indicam que a maior absorção em água por 2 horas tende para quantidades de resíduos menores. 
O tratamento estatístico para absorção em 24 horas mostrou que as variáveis de processo: resíduo e resina foram significativas no nível de $95 \%$ de confiança, com grau de correlação de $\mathrm{R}^{2}=0,99$. As curvas de nível (Figura $2 \mathrm{~b}$ ) indicam que amostras com quantidades menores de resíduo e resina absorvem maior quantidade de água, quando comparadas a outros intervalos estudados.

Esses resultados confirmam o que Vital et al. ${ }^{[29]}$ concluíram em seu trabalho, ou seja, painéis com menor razão de compactação tendem a absorver mais água devido à maior quantidade de poros e menor quantidade de material lenhoso.

\section{Propriedades mecânicas dos painéis}

Verificou-se que os resultados de MOR obtidos (Tabela 4) apresentam-se superiores ao mínimo recomendado pela norma ${ }^{[27]}$ que é $18,0 \mathrm{MPa}$, com exceção dos painéis de $1000 \mathrm{~g}$ a $10 \%$, que não atenderam ao valor mínimo especificado, apresentando resultados de 14,3 MPa. Os resultados estatísticos indicaram que as variáveis de processo resíduo e resina foram significativas. As curvas de nível para MOR (Figura 3a) indicam que há tendência para maior MOR em painéis com maior quantidade de resina, resíduo e consequentemente em painéis com maior RC.

A norma ${ }^{[27]}$ não cita especificação para MOE. Comparando os resultados com a norma estrangeira americana $^{[30]}$, verifica-se que somente os painéis de 1300 e $1500 \mathrm{~g}$ apresentaram valores acima da especificação mínima exigida por esta norma, que é de $2400 \mathrm{MPa}$. Os dados estatísticos para a variável MOE demonstraram que as variáveis resíduo e resina foram significantes ao nível de $95 \%$. A representação na forma de curvas de nível (Figura 3b) indica que para maiores quantidades de resíduos, maiores serão os resultados no $\mathrm{MOE}$, nos níveis estudados. Os resultados de MOE encontrados foram próximos aos apresentados por Iwakiri et al. ${ }^{[28]}$, com painéis da espécie Piquiarana.

Não há especificação normativa ${ }^{[27]}$ para determinação do Arrancamento de Parafuso na Face com a espessura do painel estudado $(10 \mathrm{~mm})$, porém os resultados foram superiores ao mínimo recomendado para painéis com espessuras maiores $(1020,0 \mathrm{~N})$, conforme Tabela 4, com exceção para os painéis a $1000 \mathrm{~g}$ e $10 \%$, que apresentou valor de $437 \mathrm{~N}$.

Os resultados do tratamento estatístico foram significativos $(\mathrm{p}<0,05)$ para essas condições de processo com grau de correlação de $\mathrm{R}^{2}=0,99$. As curvas de nível (Figura 3c) mostram que valores mais altos de Arrancamento de Parafuso na Face tendem para quantidades de resíduos e resinas maiores, ou seja, empregando-se a porcentagem de resina a $15 \%$ e mantendo-se a quantidade de resíduos em $1500 \mathrm{~g}$.

Os ensaios para determinação da resistência à tração perpendicular às faces, usualmente denominado de "Adesão interna" (Tabela 4) apresentaram bons resultados, cujos valores foram superiores ao mínimo $(0,40 \mathrm{MPa})$ recomendado pela norma ${ }^{[27]}$ variando de 1,71 a 1,92 $\mathrm{MPa}$.

O tratamento estatístico indicou que as variáveis estudadas não foram significativas $(p>0,05)$ neste processo. As curvas de nível (Figura 3d) mostram que

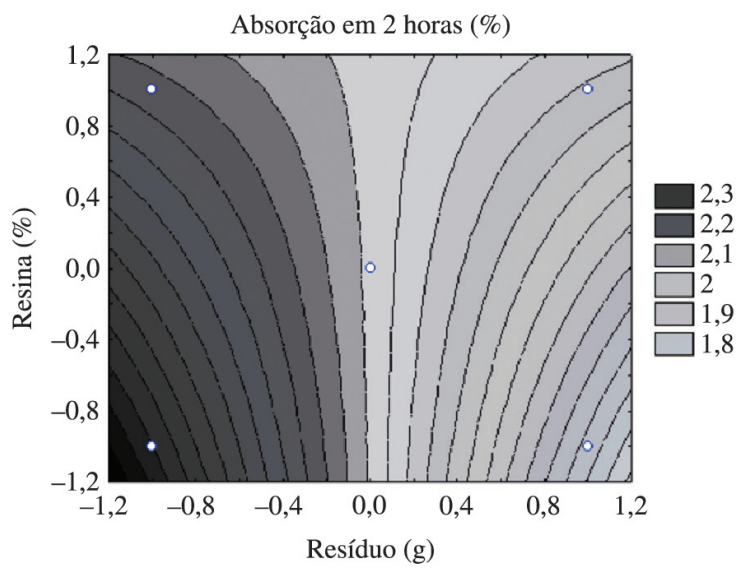

(a)

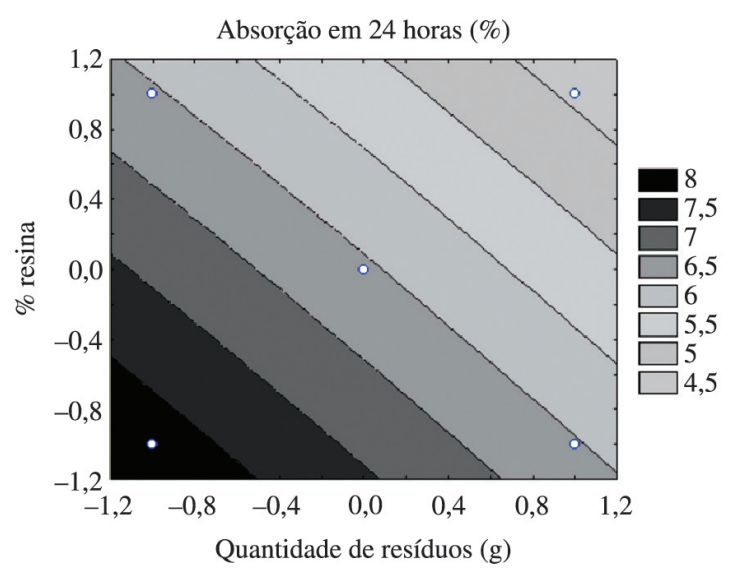

(b)

Figura 2. Curvas de nível para a variável resposta absorção em painéis de Cumaru (a, b). Quantidade de resíduos: $(-1=1000$ gramas), $(0=1300$ gramas $),(+1=1500$ gramas $) . \%$ Resina: $(-1=10 \%),(0=12,5 \%),(+1=15 \%)$.

Tabela 4. Valores médios de Módulo de Ruptura (MPa), Módulo de Elasticidade (MPa), Arrancamento de Parafuso na face (N) e Adesão Interna (MPa).

\begin{tabular}{ccccc}
\hline Condições de Processo & $\begin{array}{c}\text { Módulo de Ruptura } \\
\text { (MOR) }\end{array}$ & $\begin{array}{c}\text { Módulo de } \\
\text { Elasticidade (MOE) }\end{array}$ & $\begin{array}{c}\text { Arrancamento de } \\
\text { Parafuso na Face (N) }\end{array}$ & $\begin{array}{c}\text { Adesão Interna } \\
\text { (MPa) }\end{array}$ \\
\hline 1000 & Resina (\%) & 14,3 & 1933 & 437 \\
1000 & 10 & 19,5 & 2174 & 1050 \\
1300 & 15 & $23,6 \pm 3,03$ & $2712 \pm 347,0$ & 1,73 \\
1500 & 12,5 & 20,2 & 2709 & 2025 \\
1500 & 10 & 28,3 & 2987 & $1,92 \pm 0,35$ \\
\hline
\end{tabular}




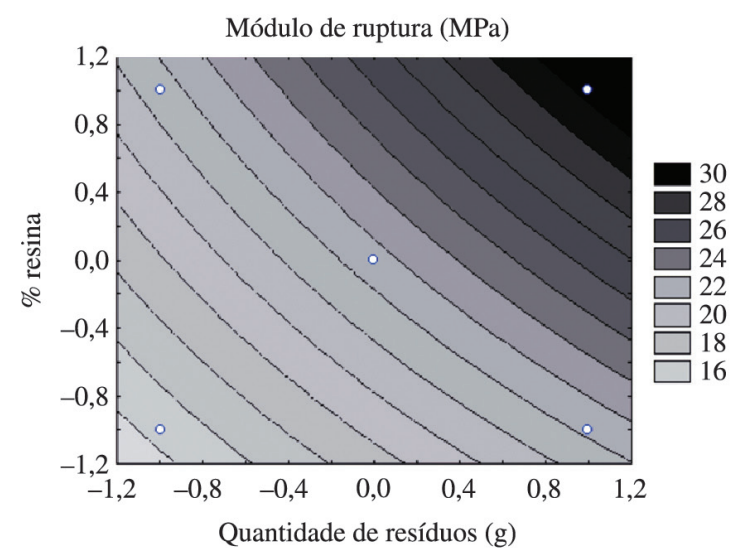

(a)

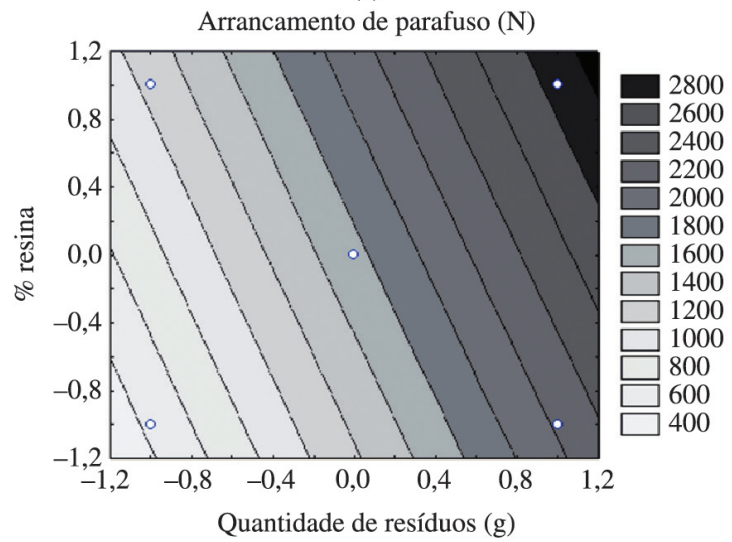

(c)

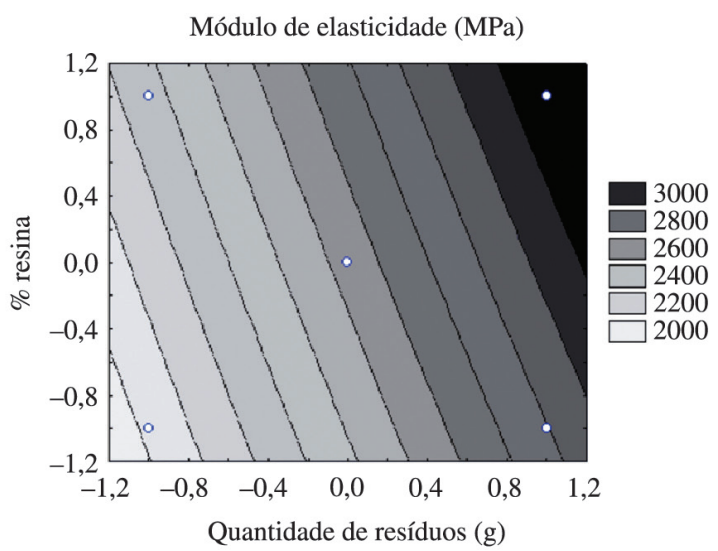

(b)

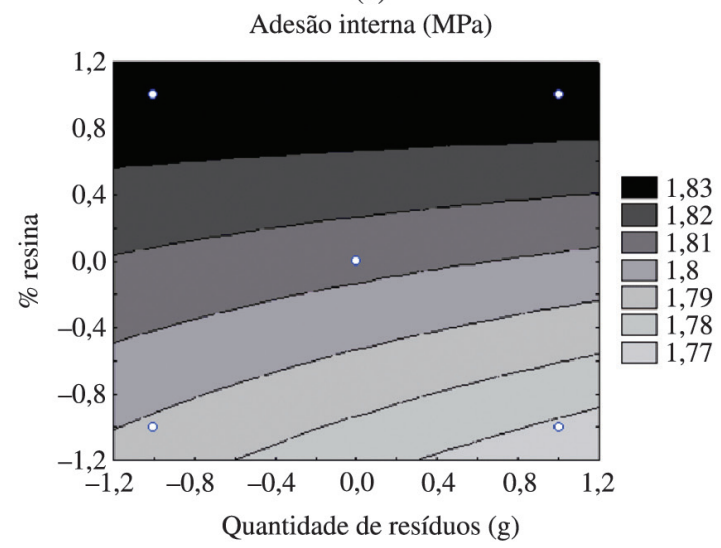

(d)

Figura 3. Curvas de nível para a variável resposta MOR (a), MOE (b), Arrancamento de Parafuso na Face (c) e Adesão Interna (d). Quantidade de resíduos: $(-1=1000$ gramas), $(0=1300$ gramas $),(+1=1500$ gramas $) . \%$ Resina: $(-1=10 \%),(0=12,5 \%),(+1=15 \%)$.

existe uma tendência para valores mais altos de Adesão Interna a maiores porcentagens de resina. Isso indica que a porcentagem de resina utilizada no intervalo de níveis investigados teve influência sobre a Adesão interna, porém não foi significativa estatisticamente. Esses resultados apresentaram-se superiores a Santos ${ }^{[7]}$ utilizando painéis de Curauá e resíduo de louro-inhamuí com média de 1,19 MPa.

\section{Conclusões}

A secagem realizada nos resíduos de Cumaru antes da fabricação dos painéis contribuiu para uma boa formação do painel e seu Teor de Umidade situou-se próximo ao recomendado para este tipo de compósito. A Densidade Básica apresentou valor característico de madeira de alta densidade confirmando os dados obtidos pela literatura sobre o Cumaru. O Teor de Cinzas e Extrativos foram superiores aos obtidos anteriormente por outros autores. O Teor de Lignina não foi significativo a ponto de prejudicar a adesão entre a madeira e a resina. O Teor de Celulose analisado apresentou valor de acordo com a literatura, com exceção das polioses que apresentaram valores mais baixos.

Verificou-se no tratamento estatístico que o resíduo é um fator significativo ( $95 \%$ de confiança) para as variáveis respostas Densidade Aparente e Razão de Compactação e que há um aumento dessas variáveis em painéis com maiores quantidades de resíduo e resina.

Valores mais altos de Inchamento se devem provavelmente à menor densidade dos painéis. $\mathrm{O}$ baixo Teor de Umidade foi verificado em quantidades maiores de resina, isto é devido às características hidrofóbicas da resina poliuretana da mamona. A maior tendência de Absorção em Água ocorreu em painéis com baixa Razão de Compactação, comprovando a tendência de maior absorção em painéis com menor quantidade de material lenhoso.

Os valores de MOR obtidos apresentaram-se superiores às exigências da norma brasileira, com exceção dos painéis produzidos com $1000 \mathrm{~g}$ a $10 \%$ e maiores valores foram obtidos em painéis com maior Razão de Compactação. Os valores de MOE e Arrancamento de Parafuso na Face apresentaram-se satisfatórios, quando comparados à outra espécie tropical, a Piquiarana. Os resultados de Adesão Interna apresentaram-se acima dos valores normativos, porém o tratamento estatístico indicou que os fatores (resíduo, resina e interação) não foram significativos considerando os níveis estudados.

A qualidade dos painéis produzidos neste estudo com o resíduo de Cumaru e a resina poliuretana à base de óleo de mamona, evidencia o potencial uso deste material na 
produção de painéis aglomerados, contribuindo para o meio ambiente através do reaproveitamento de resíduos gerados durante o processamento de madeira.

\section{Referências Bibliográficas}

1. Feitosa, B. C. - "Aproveitamento dos resíduos de madeira como alternativa para minimização de problemas sócio-ambientais no estado do Pará". Disponível em: <http://www.webartigos. com>. Acesso em: 15 fev. 2012.

2. Pinto, A. M. \& Morellato, L. P. C. \& Barbosa, A. P. - Acta Amazon., 38, p.643 (2008). http://dx.doi.org/10.1590/S004459672008000400006

3. Akyuz, K. C.; Nemli, G. \& Baharoglu, M. \& Zekoviç, E”. - Int. J. Adhes. Adhes., 30, p.166 (2010).

4. Iwakiri, S.; Zeller, F.; Pinto, J. A.; Ramirez, M. G. L. \& Souza, M. M. \& Seixas, R. - Acta Amazon., 40, p.303 (2010). http:// dx.doi.org/10.1590/S0044-59672010000200008.

5. Dias, F. M. - "Aplicação de resina poliuretana à base de mamona na fabricação de painéis de madeira compensada e aglomerada", Tese de doutorado, Universidade de São Paulo, Brasil (2005).

6. Carlo, E. \& Polito, L. W. "Desenvolvimento e caracterização de um poliuretano monocomponente baseado em óleo vegetal curado ao ar". Dissertação de Mestrado, Universidade de São Paulo, Brasil (2002)

7. Santos, F. R. S. - "Desenvolvimento e aplicação de compósitos à base de matriz polimérica reforçado com fibras de curauá (Ananás erectifolius) e resíduos de madeiras amazônicas". Dissertação de mestrado, Universidade Federal do Amazonas, Brasil (2013).

8. Mothé, C. G. \& Araújo, C. R. - Polímeros - Ciência e Tecnologia., 14, p.274 (2004)

9. Bertolini, M. S.; Lahr, F. A. R.; Nascimento, M. F. \& Agnelli, J. A. M. - Mater. Res., 16, p.293 (2013). http://dx.doi. org/10.1590/S1516-14392013005000003.

10. Goldschimid, O. - Ultraviolet spectra. In: "Lignins: occurrence, formation, structure and reactions". K. V. Sarkanen and C. H Ludwig, (eds.), John Wiley Interprice, New York (1971).

11. Novo, L. P.; Gurgel, L. V. A.; Marabezi, K. \& Curvelo, A. A. S. - Bioresour. Technol., 102, p.10040 (2011). http://dx.doi. org/10.1016/j.biortech.2011.08.050. PMid:21906937

12. Associação Brasileira de Normas Técnicas-ABNT - "Chapas de madeira aglomerada. Parte 3: Métodos de ensaio”, Rio de Janeiro (2006) (NBR 14810-3).

13. Neto, B. N.; Scarminio, I. S. \& Bruns, R. E. - "Planejamento e Otimização de Experimentos", Unicamp, Campinas (1995).

14. Nascimento, M. F. - "CPH: Chapas de Partículas homogêneas - Madeiras do Nordeste do Brasil", Tese de doutorado, Universidade de São Paulo, Brasil (2003).

15. Dias, F. M. - "Aplicação de resina poliuretana à base de mamona na fabricação de painéis de madeira compensada e aglomerada", Tese de doutorado, Universidade de São Paulo, Brasil (2005).

16. Maloney, T. M. - "Modern particleboard \& Dry-process fiberboard manufacturing”, Miller Freeman, São Francisco (1989).

17. Gonzaga, A. L. - Madeira: Uso e Conservação. Programa Monumenta (2006). Disponível em: $<$ http://www.monumenta. gov.br/upload/Caderno\%20Madeiraweb_1173383037.pdf>. Acesso em: 18 out. 2011.

18. Silva, G. A. C.; Varejão, M. J. C. \& Nascimento, C. C. "Estudos tecnológicos de alternativas de uso de resíduos madeireiros", in: Anais do 61a Reunião Anual da SBPC, p.5356, Manaus-AM (2009).

19. Santana, M. A. E. \& Okino, E. Y. A. - "Chemical composition of 36 Brazilian Amazon forest wood species", Holzforschung, Berlin (2007).

20. Lima, C. K. P.; Mori, F. A.; Mendes, L. M. \& Carneiro, A. C. O. - Cerne., 13, p.123 (2007).

21. Tienne, D. L. C. - "Qualidade da adesão de juntas coladas expostas a condições de serviço externo e interno", Dissertação de mestrado, Universidade Federal Rural do Rio de Janeiro, Brasil (2006).

22. Hillig, E. - "Qualidade de chapas aglomeradas estruturais, fabricadas com madeiras de Pinus, Eucalipto e Acácia Negra, puras ou misturadas, coladas com tanino formaldeido", Dissertação de mestrado, Universidade Federal de Santa Maria, Brasil (2000).

23. Gouvêa, A. F. G. - "Produção de briquete a partir da adição da lignina kraft com resíduo da indústria moveleira", Tese de doutorado, Universidade Federal de Viçosa, Brasil (2012).

24. Klock, U.; Muñiz, G. I. B.; Hernandez, J. A. \& Andrade, A. S. - "Química da Madeira", FUPEF, Curitiba (2005).

25. Moslemi, A. A. - "Particleboard: materials", Southern University Press, Londres (1974).

26. Maloney, T. M. - "Modern particleboard and Dry-process fiberboard manufacturing”, Miller Freeman, São Francisco (1993).

27. Associação Brasileira de Normas Técnicas-ABNT - "Chapas de madeira aglomerada. Parte 2: Requisitos", Rio de Janeiro (2006). (NBR 14810-2)

28. Iwakiri, S.; Vianez, B. F.; Weber, C. \& Trianoski, R. \& Almeida, V. C. - Acta Amazon., 42, p.59 (2012). http://dx.doi. org/10.1590/S0044-59672012000100007.

29. Vital, B. R.; Lehmann, W. F. \& Boone, R. S. - Forest Prod. Journal., 24, p.37 (1974).

30. American National Standard - ANSI - Particleboard. Gaithersburg (1999). (A208.1)

Enviado: Out. 18, 2013

Reenviado: Mar. 26, 2014 Aceito: Jun. 11, 2014 